\title{
Dealing with Inconsistencies in DL-Lite Ontologies ${ }^{\star}$
}

\author{
Liping Zhou \\ Beijing Jiaotong University, Beijing, China \\ dearliping@gmail.com
}

\section{The Research Problem}

As a shared conceptualization of a particular domain, ontologies play an important role for the success of the Semantic Web. However it is often difficult to create an absolutely consistent ontology. Inconsistency can occur due to several reasons, such as modeling errors, migration or merging ontologies, and ontology evolution. So it is essential to study how to deal with inconsistent ontologies. Many approaches have been proposed to solve this problem. These approaches are mainly used for dealing with inconsistency in expressive Description Logics(DLs). In our work, we consider inconsistency handling in the DL-Lite family, which is a family of DLs that preserve tractable reasoning and are specifically tailored to deal with large amounts of data. Like other DLs, inconsistencies in $D L$-Lite can also easily occur because disjoint axioms are allowed.

\section{The State of Art}

There are two main ways to deal with inconsistent ontologies [5]. One way is to simply avoid the inconsistency and to apply a non-standard reasoning method to obtain meaningful answers. For example, Huang et al. in [5] have proposed a general framework for reasoning with inconsistent ontologies by using a selection function. In [7812], four-valued logics have been applied to reason with inconsistent ontologies.

The other is to resolve logical modeling errors whenever a logical problem is encountered. For example, some researchers proposed methods to debug erroneous terminologies and have them repaired when inconsistencies are detected [17|18 13 16 11|4]. Some researchers considered resolving the logical inconsistencies during ontology evolution through some revision operators (see, for example, [15]).

When resolving inconsistencies, we always need some additional information to decide which solutions are better. Therefore, some researchers provided methods to measure the incoherence [14] or inconsistency [10 9]3] of ontologies. For example, some researchers provided methods for measuring inconsistency of axioms to identify which

\footnotetext{
${ }^{\star}$ I would like to acknowledge my supervisor Houkuang Huang, my co-supervisor Youli Qu, my external supervisors Zhisheng Huang and Guilin Qi for their guidance, support and fruitful discussions. Our research is supported by the National Grand Fundamental Research 973 Program of China under grant (No.2007CB307100, No.2007CB307106) and by the Specialized Research Foundation of Doctoral Program of Higher Education of China under grant (No.20050004008).
} 
axioms need to be removed or modified to resolve an inconsistency (see [3[10]). Qi and Hunter in [14] provide a method for measuring the incoherence of an ontology.

The above approaches to dealing with inconsistency are usually based on expressive DLs which suffer from worst-case exponential time behavior of reasoning [1]. This may hinder their applications to very large real life ontologies.

As a family of tractable DLs, DL-Lite can keep all the reasoning tasks tractable, in particular, with polynomial time complexity with respect to the size of the knowledge base [2]. Recently, there are some researchers began to study the problem of dealing with inconsistent $D L$-Lite ontologies. For example, Lembo et al. in [6] provided an approach for consistent query answering over ontologies specified in DL-Lite. Ma et al. in [8] proposed four-valued semantics for DL-Lite.

\section{Proposed Approach}

In our work, we mainly focus on how to handle inconsistency in DL-Lite. We will explore the following approaches. The list of approaches is not exhausting and new approach may be considered. First, we will work on a method for debugging a DL-Lite ontology by exploring the specific feature of DL-Lite. Second, we will work on a method for consistent query answering in DL-Lite based on multi-valued semantics. Third, we will propose a novel method for measuring inconsistency in a DL-Lite ontology. The main expected results of our research are as follows:

1. To give a method for measuring the inconsistency degree of $D L$-Lite ontologies. The inconsistency measures can be used to evaluate the quality of the ontology, to rank axioms in an ontology and to give guidance to resolve inconsistency. We expect that the inconsistency degree of an ontology is a single value. Unlike the work given in [10], our work uses a single value as the inconsistency degree of an ontology. Arguably, this single value is easier to be used to deal with inconsistencies, we will find an algorithm to compute the inconsistency degree in polynomial time.

2. To find a debugging algorithm tailored for DL-Lite ontologies. We will explore the tracing techniques for consistency checking algorithm in DL-Lite.

3. To design algorithms for query answering over inconsistent DL-Lite ontologies. Based [6], we plan to find a solution for query answering over inconsistency $D L$ Lite ontologies based on multi-valued semantics.

4. To implement the proposed algorithm for dealing with inconsistencies in DL-Lite ontologies.

In this paper, we will elaborate the first task, i.e., measuring inconsistency in $D L$ Lite ontologies. Our work is based on a three-valued semantics. Compared with classical semantics, three-valued semantics allows for a third truth contradictory such that even an inconsistency ontology can has three-valued models. Since we only aim to analyze inconsistency, there is no need to adopt other multi-valued semantics, such as four-valued semantics which contains a fourth truth value for expressing incomplete knowledge. 


\section{Research Methodology and Results}

\subsection{Three-Valued Semantics}

Inspired by the four-valued semantics [78], we give a three-valued semantics for $D L$ Lite. Based on the three-valued semantics, the truth values about concepts or roles have three values instead of the classical two. The three truth values are true, false and contradictory, we use the symbols $t, f, \mathbb{B}$ respectively to denote them. For a given domain $\Delta$, we give each concept or role an extended truth value $\langle P, N\rangle$, where $P$ (resp., $N$ ) is the subset of $\Delta$ (for concept) or $\Delta \times \Delta$ (for role) that supports concept or role to be true (resp., false). We denote $\operatorname{proj}^{+}(\langle P, N\rangle)=P$ and $\operatorname{proj}^{-}(\langle P, N\rangle)=N$. Given a threevalued interpretation $I$, we say that $I$ satisfies a concept inclusion axiom $B \sqsubseteq C$ (resp., a role inclusion axiom $\left.R_{1} \sqsubseteq R_{2}\right)$ if $\operatorname{proj}^{+}\left(B^{I}\right) \subseteq \operatorname{proj}^{+}\left(C^{I}\right)$ (resp., $\operatorname{proj}^{+}\left(R_{1}^{I}\right) \subseteq$ $\left.\operatorname{proj}^{+}\left(R_{2}^{I}\right)\right)$. I satisfies a function assertion (Funct $Q$ ) if $\forall x, y, z,(x, y) \in \operatorname{proj}^{+}\left(Q^{I}\right) \wedge$ $(x, z) \in \operatorname{proj}^{+}\left(Q^{I}\right) \rightarrow(y, z) \in \operatorname{proj}^{+}\left((=)^{I}\right)$. Furthermore, $I$ satisfies an atomic concept assertion $C(a)$ (resp., an atomic role assertion $R(a, b)$ ) if $a^{I} \in \operatorname{proj}^{+}\left(C^{I}\right)$ (resp., $\left.\left(a^{I}, b^{I}\right) \in \operatorname{proj}^{+}\left(R^{I}\right)\right)$. A three-valued model of a $D L$-Lite ontology $\mathcal{K}$ is a three-valued interpretation $I$ which satisfies each assertion and each axiom in $\mathcal{K}$. A $D L$-Lite ontology is three-valued satisfiable (unsatisfiable) if there exists (does not exist) such a model. Furthermore, any DL-Lite ontology has the finite model property under three-valued semantics.

\subsection{Approach to Measuring Inconsistency}

As we have discussed above, we want to compute a single inconsistency degree of a $D L$ Lite ontology. We start with studying on the conflicting atomic assertions. Considering the novel property of $D L$-Lite that a $D L$-Lite knowledge base $\mathcal{K}=\langle\mathcal{T}, \mathcal{A}\rangle$ is satisfiable iff $d b(\mathcal{A})$ is a model of $\langle\operatorname{cln}(\mathcal{T}), \mathcal{A})\rangle[2]$ and a contradiction on a $D L$-Lite KB may hold only if a membership assertion in the ABox contradicts a functionality assertion or a negative inclusions (NI) implied by the closure $\operatorname{cln}(\mathcal{T})[2]$, we think that $d b(\mathcal{A})$ and $\langle\operatorname{cln}(\mathcal{T}), \mathcal{A}\rangle$ may be useful for measuring the inconsistency degree. We plan to do as follows:

To compute all membership assertions that maybe cause inconsistency. Consider a $D L$-Lite ontology $\mathcal{K}=\langle\mathcal{T}, \mathcal{A}\rangle$ without functional assertions, we find that all conflicting assertions in $\langle\operatorname{cln}(\mathcal{T}), \mathcal{A}\rangle$ obtained through a three-valued preferred model with the domain $\Delta^{d b(\mathcal{A})}$ are also in $\mathcal{A}$. Furthermore, we find that $\mathcal{K}$ will be consistent when we remove from $\mathcal{K}$ the conflict assertions of $\langle\operatorname{cln}(\mathcal{T}), \mathcal{A})\rangle$.

Now we discuss functional assertions. Consider a $D L$-Lite ontology $\mathcal{K}=\langle\mathcal{T}, \mathcal{A}\rangle$, where $\mathcal{T}$ only contains functional assertions. If $\mathcal{K}$ is inconsistent, we can know that there are some membership assertions in $\mathcal{A}$ contradicting with some functional assertions. By computing preferred models of $\mathcal{K}$ with any domain, we find that the conflict sets obtained in any preferred model with respect to different domains are the same and assertions in conflict sets are all in the form $=(a, b) 1$. For example, for $\mathcal{K}=\{(F$ unct $R), R(a, b), R(a, c)\}$, the conflict set is $\{=(b, c)\}$. We also note that the conflict set is only related with $\mathcal{A}$, so we have the following definition.

\footnotetext{
${ }^{1}$ We enforce the unique name assumption on constants [1].
} 
Definition 1 (Inconsistency Degree). Let $\mathcal{K}=\langle\mathcal{T}, \mathcal{A}\rangle$ be a DL-Lite ${ }_{\mathcal{R}}$ or a DL-Lite $\mathcal{F}$ ontology, and let I be a three-valued preferred model of $\langle\operatorname{cln}(\mathcal{T}), \mathcal{A}\rangle$ w.r.t $\Delta^{d b(\mathcal{A})}$. The inconsistency degree of DL-Lite $\mathcal{R}_{\mathcal{R}}$ ontology (resp., DL-Lite $\mathcal{F}$ ontology), called OntoInc $(\mathcal{K})$, is defined as: OntoInc $(\mathcal{K})=\frac{|\operatorname{ConSet}(I,\langle\operatorname{cln}(\mathcal{T}), \mathcal{A}\rangle)|}{|\operatorname{GroundSet}(\mathcal{K})|}(\operatorname{resp} ., \quad$ OntoInc $(\mathcal{K})$ $\left.=\frac{|\operatorname{ConSet}(I,\langle\operatorname{cln}(\mathcal{T}), \mathcal{A}\rangle)|+\operatorname{ConEqus}(I,\langle\operatorname{cln}(\mathcal{T}), \mathcal{A}\rangle)}{|\operatorname{GroundSet}(\mathcal{K})|}\right)$, where $\operatorname{ConSet}(I,\langle\operatorname{cln}(\mathcal{T}), \mathcal{A}\rangle)$ is the set of conflicting atomic individual assertions in $\langle\operatorname{cln}(\mathcal{T}), \mathcal{A}\rangle$ regardless of functional assertions and $\operatorname{Con} \operatorname{Equs}(I,\langle\operatorname{cln}(\mathcal{T}), \mathcal{A}\rangle)$ is the set of conflicting assertions obtained through functional assertions and GroundSet $(\mathcal{K})$ is the collection of all possible atomic individual assertions.

From Definition 1, we can see that the inconsistency degree is only a single value. The next step is only to compute a preferred model of $\langle\operatorname{cln}(\mathcal{T}), \mathcal{A}\rangle$ with the domain $\Delta^{d b(\mathcal{A})}$ so as to obtain $\operatorname{ConSet}(I,\langle\operatorname{cln}(\mathcal{T}), \mathcal{A}\rangle)$ and $\operatorname{ConEqus}(I,\langle\operatorname{cln}(\mathcal{T}), \mathcal{A}\rangle)$.

\subsection{Algorithm to Compute a Preferred Model of $\langle\operatorname{cln}(\mathcal{T}), \mathcal{A}\rangle$}

Using the property that $\operatorname{cln}(\mathcal{T})$ only contains NIs and functional assertions and the definition of chase in [2], we can give a definition of chase which mainly adapt to $\langle\ln (\mathcal{T}), \mathcal{A}\rangle$ which is finite. Then based on this chase, we give an algorithm to compute a three-valued model of $\langle\ln (\mathcal{T}), \mathcal{A}\rangle$ which is a preferred model of $\langle\operatorname{cln}(\mathcal{T}), \mathcal{A}\rangle$ with the domain $\Delta^{d b(\mathcal{A})}$. So we can obtain $\operatorname{Con} \operatorname{Set}(I,\langle\operatorname{cln}(\mathcal{T}), \mathcal{A}\rangle)$ and $\operatorname{ConEqus}(I$, $\langle\ln (\mathcal{T}), \mathcal{A}\rangle)$. We show that the complexity of the algorithm is in PTime. Finally, we can obtain the inconsistent degree of $\langle\mathcal{T}, \mathcal{A}\rangle$ based on Definition 1

\subsection{Evaluation}

We plan to implement the algorithm for measuring the inconsistency degree proposed above and give a prototype. Meanwhile we will give some potential applications of our tool. For example, users can input some DL-Lite ontologies and obtain the inconsistency degree of each ontology; users can either resolve inconsistency if the degree is high (e.g. greater than 0.7 [10]) or ignore it.

\section{Conclusion}

Dealing with inconsistency in ontologies is an important topic in the Semantic Web. In our paper, we mainly discuss how to handle inconsistency in DL-Lite ontologies. We first give some proposals for our work, then we illustrate how to measure inconsistency in a DL-Lite ontology based on a three-valued semantics.

\section{References}

1. Baader, F., Calvanese, D., McGuinness, D., Nardi, D., Patel-Schneider, P.: Description logic terminology. In: Baader, F., et al. (eds.) The Description Logic Handbook: Theory, Implementation, and Applications. Cambridge University Press, Cambridge (2003) 
2. Calvanese, D., De Giacomo, G., Lembo, D., Lenzerini, M., Rosati, R.: Tractable reasoning and efficient query answering in description logics: The DL-Lite family. J. Autom. Reasoning 39(3), 385-429 (2007)

3. Deng, X., Haarslev, V., Shiri, N.: Measuring inconsistencies in ontologies. In: Franconi, E., Kifer, M., May, W. (eds.) ESWC 2007. LNCS, vol. 4519, pp. 326-340. Springer, Heidelberg (2007)

4. Friedrich, G., Shchekotykhin, K.: A general diagnosis method for ontologies. In: Gil, Y., Motta, E., Benjamins, V.R., Musen, M.A. (eds.) ISWC 2005. LNCS, vol. 3729, pp. 232-246. Springer, Heidelberg (2005)

5. Huang, Z., van Harmelen, F., ten Teije, A.: Reasoning with inconsistent ontologies. In: Kaelbling, L.P., Saffiotti, A. (eds.) Proceedings of the 19th International Joint Conference on Artificial Intelligence, pp. 454-459 (2005)

6. Lembo, D., Ruzzi, M.: Consistent query answering over description logic ontologies. In: Marchiori, M., Pan, J.Z., de Sainte Marie, C. (eds.) RR 2007. LNCS, vol. 4524, pp. 194-208. Springer, Heidelberg (2007)

7. Ma, Y., Hitzler, P., Lin, Z.: Algorithms for paraconsistent reasoning with OWL. In: Franconi, E., Kifer, M., May, W. (eds.) ESWC 2007. LNCS, vol. 4519, pp. 399-413. Springer, Heidelberg (2007)

8. Ma, Y., Hitzler, P., Lin, Z.: Paraconsistent reasoning for expressive and tractable description logics. In: Baader, F., Lutz, C., Motik, B. (eds.) Description Logics (2008)

9. Ma, Y., Qi, G., Hitzler, P., Lin, Z.: An algorithm for computing inconsistency measurement by paraconsistent semantics. In: Mellouli, K. (ed.) ECSQARU 2007. LNCS, vol. 4724, pp. 91-102. Springer, Heidelberg (2007)

10. Ma, Y., Qi, G., Hitzler, P., Lin, Z.: Measuring inconsistency for description logics based on paraconsistent semantics. In: Mellouli, K. (ed.) ECSQARU 2007. LNCS, vol. 4724, pp. 30-41. Springer, Heidelberg (2007)

11. Parsia, B., Sirin, E., Kalyanpur, A.: Debugging OWL ontologies. In: Ellis, A., Hagino, T. (eds.) Proceedings of the 14th International World Wide Web Conference, pp. 633-640 (2005)

12. Patel-Schneider, P.F.: A four-valued semantics for terminological logics. Artif. Intell. (3), 319-351 (1989)

13. Qi, G., Haase, P., Huang, Z., Ji, Q., Pan, J.Z., Völker, J.: A kernel revision operator for terminologies - algorithms and evaluation. In: Sheth, A.P., et al. (eds.) ISWC 2008. LNCS, vol. 5318, pp. 419-434. Springer, Heidelberg (2008)

14. Qi, G., Hunter, A.: Measuring incoherence in description logic-based ontologies. In: Aberer, K., Choi, K.-S., Noy, N., Allemang, D., Lee, K.-I., Nixon, L., Golbeck, J., Mika, P., Maynard, D., Mizoguchi, R., Schreiber, G., Cudré-Mauroux, P. (eds.) ASWC 2007 and ISWC 2007. LNCS, vol. 4825, pp. 381-394. Springer, Heidelberg (2007)

15. Qi, G., Liu, W., Bell, D.A.: Knowledge base revision in description logics. In: Fisher, M., van der Hoek, W., Konev, B., Lisitsa, A. (eds.) JELIA 2006. LNCS, vol. 4160, pp. 386-398. Springer, Heidelberg (2006)

16. Schlobach, S.: Diagnosing terminologies. In: Veloso, M.M., Kambhampati, S. (eds.) Proceedings of the 20th National Conference on Artificial Intelligence, pp. 670-675 (2005)

17. Schlobach, S., Cornet, R.: Non-standard reasoning services for the debugging of description logic terminologies. In: Gottlob, G., Walsh, T. (eds.) Proceedings of the 17th International Joint Conference on Artificial Intelligence, pp. 355-362 (2003)

18. Schlobach, S., Huang, Z., Cornet, R., van Harmelen, F.: Debugging incoherent terminologies. J. Autom. Reasoning 39(3), 317-349 (2007) 